\title{
Procesos de vinculación, permanencia y reintegración social de niños y niñas que participaron en grupos armados ilegales en Colombia*
}

\author{
Processes of Involvement, Permanence, and Social \\ Reintegration of Children Who Participated in Illegal Armed \\ Groups in Colombia
}

\author{
Cindy Marcela Melo-Rincón \\ ORCID: 0000-0002-6790-5934 \\ Ángela Victoria Vera-Márquez** \\ ORCID: 0000-0002-5829-0069 \\ Miguel Gutiérrez-Peláez \\ ORCID: 0000-0003-0816-2605 \\ Universidad del Rosario, Colombia \\ Recibido: 22 de septiembre de 2019 \\ Revisado: 5 de diciembre de 2019 \\ Aceptado: 30 de marzo de 2020
}

\section{Resumen}

El presente trabajo tuvo como objetivo realizar una revisión de la literatura sobre los procesos de vinculación, permanencia y reintegración de niños y niñas que participaron en grupos armados ilegales de Colombia (GAI). Se revisaron 51 documentos de bases de datos especializadas y catálogos de universidades colombianas en el periodo entre 2005 y 2018. Los resultados mostraron que la mayoría de los estudios realizados se concentran en los procesos de vinculación y reintegración. Se discuten diferentes perspectivas sobre la denominación de este grupo poblacional, se mencionan las causas identificadas para la vinculación, dinámicas de interacción y ajuste a los GAl durante la permanencia. Así mismo, se analizan las vivencias en los procesos de reintegración y características de los programas de atención y se presentan algunas recomendaciones para los procesos de reintegración.

Palabras clave: excombatientes, niños, grupos armados ilegales, conflicto armado, reintegración

Artículo de investigación. Citar como: Melo-Rincón, C. M., Vera-Márquez, A. V. y Gutiérrez-Peláez, M. (2020). Procesos de vinculación, permanencia y reintegración social de niños y niñas que participaron en grupos armados ilegales en Colombia. Diversitas: Perspectivas en Psicología, 16(2), 413-425. https://doi.org/10.15332/22563067.5654

Artículo elaborado con base en la investigación: Participación de niños y niñas en los grupos armados ilegales en Colombia: una revisión de la literatura de los procesos de reclutamiento, permanencia y desmovilización desde el enfoque psicosocial; para el Programa de Psicología de la Escuela de Medicina y Ciencias de la Salud, de la Universidad del Rosario. Bogotá-Colombia.

Autor de correspondencia: Ángela Victoria Vera-Márquez. Profesora de Carrera. Programa de Psicología. Escuela de Medicina y Ciencias de la Salud, GI Estudios Sociales de las Ciencias, las Tecnologías y las Profesiones, Universidad del Rosario. Bogotá, Colombia. Correo electrónico: angela.vera@urosario.edu.co. Dirección postal: Ak. 24 No 63C-69, Bogotá 


\section{Abstract}

The current work aimed to review the literature on the processes of involvement, permanence and reintegration of children who participated in illegal armed groups (IAG) in Colombia. Fifty-one (51) documents of specialized databases and catalogs of Colombian universities were reviewed in the period between 2005 and 2018. The results showed that most of the studies on the participation of children in armed groups are focused on the processes of involvement and reintegration. Different perspectives on the denomination of this population group are discussed, as well as the causes identified for the involvement and the dynamics of interaction and adjustment to the IAG during the stay. Besides, the experiences involved during the reintegration processes were analyzed and the characteristics of the care programs are mentioned. Finally, some recommendations for the reintegration process are presented.

Key words: ex-combatants, children, illegal armed groups, armed conflict, reintegration.

\section{Introducción}

La presente investigación documental consistió en una revisión de la literatura sobre la participación de niños y niñas en los grupos armados ilegales de Colombia en estudios nacionales publicados entre 2005-2018, considerando los procesos de reclutamiento-vinculación, permanencia y reintegración. El interés fue analizar la transición de los niños y las niñas excombatientes a lo largo de estos tres procesos, y visibilizar las consecuencias en el desarrollo de la infancia que ha crecido en este tipo de entornos vulnerables (Torrado, 2002).

El reclutamiento de niños, niñas y jóvenes en Colombia es un fenómeno preocupante. Se encuentran antecedentes de su participación en la confrontación armada desde los años sesenta; y según el Observatorio de Memoria y Conflicto (OMC), el reclutamiento ha sido de 16.879 niños y niñas y el promedio de ingreso formal fue de 12.9 años (Centro Nacional de Memoria Histórica - $\mathrm{CNMH}$, 2017). Su participación en grupos armados ilegales representa una vulneración a sus derechos fundamentales (Bácares, 2014; Blom y Pereda, 2009; Fajardo, Ramírez, Valencia y Ospina-Alvarado, 2018; Springer, 2012), en el que se ven afectadas diferentes dimensiones de su desarrollo social y personal $y$ aunque se han generado iniciativas que intentan protegerlos, aún persiste un desconocimiento sobre su situación (Fajardo et al., 2018; Montoya, 2008).

El análisis de las implicaciones de la participación de esta población en grupos armados es un campo que ha generado cuestionamientos sobre el papel de diversos actores tanto políticos, sociales y académicos. Los desarrollos legislativos alcanzados en Colombia siguen normativas internacionales, que involucran las condiciones propias de los niños que han sido vinculados al conflicto armado (Caicedo, 2012; Granados y Lavado, 2015). Pero, en cuanto a la producción académica, se considera que dada la complejidad, se requieren comprensiones que aporten a la construcción de políticas públicas y programas de intervención social (Cifuentes, Aguirre y Lugo, 2011; Torrado, 2002). En este marco, realizar una revisión de literatura contribuye a brindar un panorama que aporte a la comprensión de este fenómeno y permite hacer un seguimiento de la producción académica enfocada a la prevención de este fenómeno y a los procesos de reintegración.

\section{Método}

El proceso de revisión de literatura se llevó a cabo en tres momentos. En el primer momento se realizó una búsqueda bibliográfica en las bases de datos 
especializadas de Scielo, Redalyc, Google Scholar y Scopus. La búsqueda se realizó usando operadores boléanos como "Niños AND ex combatientes AND Colombia", "Infancia AND ex combatientes AND Colombia", "infancia AND soldado AND Colombia". El criterio fundamental de inclusión fue que los documentos estuvieran publicados entre los años 2005 al 2018 y que abordaran el tema de niños y niñas excombatientes de grupos armados ilegales en Colombia. Se revisaron 51 documentos: 29 empíricos, 16 trabajos de revisión y 6 reflexiones teóricas.

En el segundo momento se elaboraron resúmenes analíticos, haciendo uso de matrices de caracterización que permitieron organizar los resultados en categorías. La caracterización de los artículos se hizo de acuerdo con los siguientes criterios: año de publicación, tipo de investigación, procesos de participación de los niños y las niñas en los grupos armados que abordó el artículo, perspectiva del documento, tema o aproximación teórica, tipo de documento y zona geográfica. Además, se realizó un proceso de sistematización a través de la construcción de resúmenes analíticos especializados (RAE). Con este instrumento se logró identificar, en cada uno de los procesos, los argumentos que eran relevantes para el fenómeno de estudio. A su vez, se construyeron categorías de análisis dependiendo del tema central que abordaban.

En el tercer y último momento se realizó la integración de los resultados. En particular, para el presente artículo los hallazgos se presentarán, principalmente, en tres apartados: vinculación, permanencia y reintegración. Sin embargo, se consideró pertinente exponer dos apartados más, uno sobre las percepciones sociales sobre este grupo poblacional y otro, al final, en el que se incluyen algunas recomendaciones para los procesos de reintegración de esta población.

\section{Percepciones sociales sobre los niños y las niñas en la guerra}

Los niños y las niñas que han participado en grupos armados ilegales hacen parte de una construcción social en el que se articulan diversas dimensiones, procesos y actores (Bácares, 2017). En cuanto a denominación de este grupo social en la revisión de la

Tabla 1.

Categorías para referirse a niños, niñas y jóvenes que han estado en la guerra

\begin{tabular}{|c|c|}
\hline $\begin{array}{l}\text { Definición } \\
\text { Niños y niñas }\end{array}$ & Concepto \\
\hline Soldados & $\begin{array}{l}\text { Cualquier persona menor de } 18 \text { años que forma parte de cualquier tipo de fuerza o movimiento } \\
\text { armado, ya sea regular o irregular, en cualquier condición, incluyendo también a cocineros, } \\
\text { recaderos, mensajeros y cualquier otra persona que acompañe a dichos grupos y no sea sola- } \\
\text { mente un familiar. No se refiere solo al uso de un arma (Unicef, } 1997 \text { citado por Blom y Pereda, } \\
2009, \text { p.330). }\end{array}$ \\
\hline Excombatientes & $\begin{array}{l}\text { Reconoce el estatus de aquel que fue combatiente -que es importante en la definición que } \\
\text { ellos hacen de sí mismos- y evita denominaciones descalificadoras como terroristas, rebeldes } \\
\text { o bandidos. El combatiente fue aquel que tomó las armas como parte de un grupo implicado en } \\
\text { un conflicto armado. Significa también no perder del todo la posición en el orden social que los } \\
\text { jóvenes alcanzaron como combatientes, que les otorgó reconocimiento y respeto, el prestigio } \\
\text { del guerrero (Lugo, 2018, p. 18) }\end{array}$ \\
\hline Desvinculado & $\begin{array}{l}\text { Es todo menor de edad que ha participado en acciones bélicas durante el conflicto armado y ha } \\
\text { pertenecido a un grupo armado irregular (Chamorro, 2012, p.128). Es la manera legal, oficial y } \\
\text { común de nombrar a estos niños y jóvenes en Colombia (Lugo, 2018, p.18) }\end{array}$ \\
\hline Desmovilizado & $\begin{array}{l}\text { Hace referencia a todos los mayores de edad que integraban las filas armadas (Chamorro, } 2012 . \\
\text { p.128) }\end{array}$ \\
\hline
\end{tabular}

Fuente: elaboración propia. 
literatura se encuentra que los investigadores proponen diferentes acepciones, refiriéndose a ellos como niños y niñas: "soldados", "excombatientes", "desvinculados" y "desmovilizados". Frente a cada una de estas categorías se presentan algunas precisiones sobre las definiciones y sobre cuándo nos referiremos a cada una de ellas (ver tabla 1).

Frente a estos diferentes usos del lenguaje para referirse a la población, Lara y Delgado (2010) consideran que dichas formas expresan imaginarios colectivos y pueden incidir en la determinación de identidades. Los autores sugieren que sean denominados niños, niñas y jóvenes que estuvieron en la guerra y han indicado, por ejemplo

[...] que no podemos seguir hablando de 'vinculados', cuando hay sujetos que nunca se vincularon al conflicto armado; de 'desvinculados', cuando hay sujetos que, a pesar de estar en el contexto de la vida civil, siguen vinculados emocionalmente con el grupo armado (Lara y Delgado, 2010, p.54).

De ahí que sugieren sean revisadas las categorías y se reinterpreten para que se aproximen más a la realidad de esta población.

Algunas investigaciones, como las de Niño (2016) y Correa y colaboradores (2014), encontraron una visión dicotómica de la infancia, que ha participado en los GAl, que los ubica entre la perspectiva de "víctima" y "victimario" o "criminales". Estas miradas se han corroborado en estudios que han indagado sobre las percepciones de actores académicos (Moreno, Carmona y Tobón, 2010), niños antioqueños (Carmona y Tobón, 2007), estudiantes, docentes y policías (Carmona, Moreno y Tobón, 2012) sobre por qué las niñas se vinculan a los GAl. Estos trabajos han encontrado un predominio de factores deterministas (por ejemplo, violencia, la pobreza) cuando representan a las niñas excombatientes como "víctimas" y argumentos no deterministas cuando se relaciona con supuestos deseos criminales por parte de éstas (Carmona y Tobón, 2007). En la línea de las percepciones de los niños desvinculados a los GAl como víctimas, algunos autores resaltan las afectaciones que estos viven en la guerra, traduciéndose en vulneraciones a sus derechos (Bácares, 2014; Springer; 2012; Valencia-Suescún, Ramírez,
Fajardo y Ospina-Alvarado, 2015). Bácares (2015) plantea que el pronunciamiento en las leyes 418 de 1997, 782 de 2002 y en la 1448 de 2011, que considera a esta población como víctimas, es un acto loable, que se articula con una postura humanitaria que ubica el reclutamiento al nivel de un delito y de un crimen de guerra.

En esta misma línea, Velasco y García (2013) expresaron que esta comprensión no implica subvalorarlos, sino reconocer la responsabilidad del Estado y el impacto que ha generado el conflicto armado sobre ellos. Adicionalmente, es permitirles ejercer una ciudadanía e identidad que les fue arrebatada (Velasco y García, 2013). Al respecto, Pinto (2009) expone que, aunque debe reconocerse la condición de víctima del reclutamiento armado, es importante no victimizarlos a partir de esta circunstancia.

En relación con las percepciones sociales, Nabuco y Duarte (2018) exponen que en la mayoría de los casos no tienen en cuenta el papel "agente" de los niños, niñas y adolescentes en los esfuerzos de construcción de la paz. Los autores realizaron un análisis crítico del discurso de cómo fueron clasificados y representados en el acuerdo de paz del gobierno colombiano y las FARC en el 2017 e identificaron que fueron categorizados con una visión ambigua, como víctimas y perpetradores con posibilidades para participar en la construcción de la paz, pero a la vez, fueron considerados como una población que representa potenciales desafíos para el proceso, principalmente porque pueden ser una amenaza para la paz por el riesgo de que sean reclutados de nuevo.

\section{Vinculación a los grupos armados ilegales}

En el proceso de vinculación se describen las posibles causas que conducen al ingreso de los niños y las niñas a los GAl. En este proceso se debe considerar su dinámica histórica, ya que no es lo mismo hablar del periodo de 1978 - 1989, momento en el que empieza en el país el reclutamiento de menores dada la necesidad de ampliar las filas de los actores armados, que hacerlo entre 2005 - 2015, en donde ocurren los procesos de desmovilización de ciertos actores como las Autodefensas Unidas de Colombia 
y las faRC-ep (CNMH, 2017). En este contexto, de la revisión de la literatura se han extraído diferentes factores que pueden conllevar a la vinculación a los GAl, los cuales involucran aspectos contextuales, familiares, personales y de género.

Como aspectos contextuales, algunas investigaciones han señalado la pobreza y la ausencia del Estado y la presencia de los GAl en el entorno (Bjørkhaug, 2010; Downing, 2014, Rojas, 2014; Springer, 2012). Frente a esto, Vargas y Restrepo-Jaramillo (2016) encontraron que el contexto sí es importante en el reclutamiento ya que los municipios con las mayores altas tasas de pobreza rural, presencia de cultivos ilícitos y falta de acceso a la educación tienen más probabilidades de ser zonas de reclutamiento por los GAl.

En consonancia con esto, Amador-Baquiro (2010) y Pinto (2009) identificaron en sus estudios que los escenarios sociales, así como las prácticas culturales (Pieschacón, Melguizo y González, 2006) en los que nacen y crecen los niños y las niñas vinculadas a los GAl, favorecen marcos de violencia que puede convertirse en sus propios referentes. Molina (2011) identificó en un programa de jóvenes desmovilizados en el que la mayoría se caracterizaban "por su bajo nivel de escolaridad, por provenir de familias pobres y de zonas con presencia histórica de un actor armado o en disputa por dos actores armados" (p.182).

Aunque en los antecedentes se hace mención de que no siempre la vinculación es obligada, Rojas (2014) identificó que esto es relativo ya que muchos niños en sus contextos no tienen otra oportunidad laboral que la opción de vinculación a los GAl. Adicionalmente, Pieschacón y colaboradores (2006) identificaron otras categorías como ser víctima de desplazamiento forzado, ser niño en situación de trabajo infantil y no tener opciones para la educación.

El funcionamiento familiar puede llegar a ser un facilitador del reclutamiento (Downing, 2014; Pieschacón et al., 2006). Torres (2015) encontró que para algunos niños la familia se ha desdibujado debido a las experiencias de exclusión, maltrato y discriminación en sus hogares. Investigaciones con mujeres desmovilizadas (Ocampo, Baracaldo,
Arboleda y Escobar, 2014) y con niñas desvinculadas (Chamorro, 2012) han identificado la falta de cuidado, falta de protección, abuso sexual y maltrato. En esta línea, la violencia intrafamiliar es una variable relevante, debido a que en algunas niñas la vinculación al grupo guerrillero representó una forma de huir de esa situación (Carmona 2013; Moreno, Carmona y Tobón, 2010), buscar un nuevo lugar en la estructura familiar para influir en su dinámica (Moreno, Carmona y Tobón, 2010) y ganar respeto y reconocimiento (Ocampo et al., 2014). Con esto, lograron protegerse frente al maltrato de algún miembro de su familia (Chamorro, 2012) y se convirtió en una posibilidad de abandonar una situación de marginalización (Bjørkhaug, 2010).

Pinto (2009) expone que una infancia caracterizada por el maltrato y vínculos afectivos débiles se convierte en un factor de vulnerabilidad ya que favorecen relaciones basadas en la desconfianza. Estos aspectos propician la huida de sus familias y el refugio en las figuras de poder, como las que ofrecen los Gal (Garcés y Chamarro, 2011). Sin embargo, en las causas de dominio personal, autores como Moreno, Carmona y Tobón (2010) han encontrado que para las niñas que han estado vinculados a los grupos armados, la institucionalización de la violencia pasa como proceso inadvertido y buscan explicaciones centradas en sus biografías.

Aunque algunos excombatientes de los GAl han reportado que fueron forzados a pertenecer a los grupos armados (Downing, 2014), la mayoría han declarado que se unieron de forma voluntaria (Bjørkhaug, 2010; Vargas y Restrepo-Jaramillo, 2016) y progresiva, de modo que no representó mayores traumatismos (Amador-Baquiro, 2010). Algunos niños, niñas y jóvenes, al reconstruir su experiencia consideran que su vinculación se dio como opción política y por ansías de libertad, autonomía o reconocimiento (Pieschacón et al., 2006). Moreno, Carmona y Tobón (2010) identificaron en niñas excombatientes argumentos de vinculación al grupo armado relacionados con aspiraciones lúdicas (45\%) como la "aventura", en el sentido de conocer nuevos lugares y personas, tener vestimenta militar como un atuendo llamativo y la "diversión" relacionada con la imagen de fiestas, interacciones y el coqueteo (p.462). 
Dentro de los motivos de vinculación explorados, se identificaron diferencias de género. Así pues, para las niñas excombatientes, la decisión estuvo mediada por la importancia de las relaciones interpersonales cercanas, las necesidades afectivas y a sus aspiraciones (Bjørkhaug, 2010; Carmona, 2013; Carmona y Tobón, 2007). Al respecto, Moreno, Carmona y Tobón (2010) identificaron motivos relacionados con aspectos, tales como el enamoramiento $(10 \%)$ y el amor filial (10\%), en un menor caso se presentaron en las narraciones, deseos de venganza (5\%). Por su parte, en los niños se ha encontrado mayor peso el deseo de poder (Moreno, Carmona y Tobón, 2010) y de estatus (Downing, 2014).

Finalmente, algunos análisis integradores han identificado que en el conflicto Colombiano, independientemente del grupo armado, los motivos de vinculación comprenden tres niveles: factores relacionados con el contexto en el que crece el combatiente, historia personal que puede predisponer a los jóvenes a ingresar y desencadenantes de la decisión específica de vinculación (Valencia y Daza, 2010).

\section{Permanencia en los grupos armados ilegales}

La permanencia en los Gal es un proceso complejo que implica una serie de transformaciones, ritmos y metamorfosis para el nuevo estilo de vida "como guerreros" (Torres, 2015) que debe ser entendida como una experiencia múltiple y no necesariamente marcada por traumas (Vera, 2017). Los estudios que abordan las experiencias de menores en los GAl señalan aspectos como: dinámicas en las interacciones entre los GAl y los niños para adaptarse al nuevo escenario, diferencias en las interacciones de acuerdo con el género, razonamientos de los niños frente a situaciones morales y valoraciones sobre las afectaciones que genera este tipo de vivencias en los actores implicados.

Los menores que participaron en el conflicto armado, una vez inmersos en los GAl, son sometidos a un modelo de disciplina (Bjørkhaug, 2010; Sánchez, Rojas y Taborda, 2015). En este proceso, se requieren cuerpos aptos para el combate, pero a la vez, dóciles para que cumplan órdenes sin mayor objeción (Sánchez, Rojas y Taborda, 2015). En estas dinámicas en las que se exponen continuamente a situaciones de maltrato, también encuentran la posibilidad de ejercer dominio frente a otros "enemigos o más débiles" (Amador-Baquiro, 2010) y se transforman de víctimas a victimarios (AmadorBaquiro, 2010, Torres, 2015).

El proceso de formación como parte de los GAl ha llevado a los menores a situaciones en las que no solo ha estado en riesgo su vida, sino también en la que han debido decidir sobre la vida de otras personas y en las que han instalado nuevas lógicas para adaptarse a la vida militar y aprender a combatir, a sobrevivir y a utilizar armas (Amador-Baquiro, 2010, Vera, 2017). También, se ha encontrado que pueden asumir relaciones de poder y alianzas con sus compañeros combatientes como una forma de supervivencia (Garcés y Chamorro, 2011). En ocasiones, llegan a considerar a los miembros de sus grupos como su familia (Vera, 2017), aunque no logren con esto subsanar las deudas afectivas con sus familias de origen (Garcés y Chamorro, 2011).

Durante la permanencia en el grupo armado, Vera (2017) identificó, en las narrativas, dilemas morales cuando narraban situaciones que implicaban infringir daño a otros. Aunque reconocían las implicaciones morales y emocionales de lastimar a otros (ej. matar), justificaban sus acciones desde las consecuencias que debían asumir si infringían las reglas $y$, de no hacerlo, se pondría en entredicho su bienestar personal y el del grupo. Aun así, valoraban como negativa la acción, y reconocían que de no ser por la obligatoriedad que se desprende de estos actos, hubiesen preferido negarse a actuar de esta forma. Al respecto, Wainryb (2011) identificó que los niños en estas circunstancias intentan conciliar sus propias acciones con una visión de sí mismos como personas morales. La autora encontró que esta población construye de forma problemática su sentido de agencia moral, estando esta entumecida, dividida o restringida externamente.

En el marco de los GAl, Chamorro (2012) identificó que se generan relaciones de poder que tiene matices diferentes dependiendo del género. En el caso de las mujeres, hay un control de su cuerpo y de su sexualidad $y$, aunque al ingresar al grupo armado logran ejercer un poder hacia fuera, en el grupo 
siguen estando sujetas a las órdenes de otros. En el caso de niños y hombres también afectados, estar en el grupo los orienta a la búsqueda de poder, su reconocimiento en lo público y la posibilidad de someter a otros (Chamarro, 2012). Para Chamorro (2012), las dinámicas de poder que se desarrollan en los Gal contradicen los sentimientos, roles y prácticas de los niños y las niñas, cuestionando sus formas de ser hombre o mujer y de ser aceptados en la sociedad. En el caso de las mujeres, la autora comentó que, si bien pueden ingresar para proteger a otros o protegerse, luego, frente a las dinámicas de violencia del GAl, silencian sus discursos de reivindicación de su rol como una forma de sobrevivir.

Las experiencias violentas que enfrentan en los GAl, generan múltiples heridas, no solo en el cuerpo, sino también en las subjetividades (Sánchez et al., 2015). Además, las vivencias en el conflicto armado son sentidas no solo en el grupo armado que representan, sino también entre los sujetos heridos y los seres más próximos como familiares y amigos. En este proceso es que los investigadores Sánchez, Rojas y Taborda (2015) consideran que todos los actores experimentan heridas y algunos de ellos, cicatrices.

\section{Proceso de reintegración de niños y niñas excombatientes}

La reintegración es considerada como la etapa final del proceso de DDR. En esta etapa se debe garantizar que los niños y las niñas que se han desmovilizado no regresen a la violencia y, también que puedan trabajar en la construcción de vínculos de confianza con las comunidades (Mesa, 2017). Esto significa que los niños, las niñas y los jóvenes que se han desmovilizado de los GAl, y hacen una transición hacia la vida civil, tienen la oportunidad de dar sus relatos del pasado, del presente y sus proyectos futuros (Torres, 2015). En la presente revisión, se identificaron investigaciones en situaciones referidas a la adaptación social, cuestiones afectivas, procesos identitarios y dificultades en los programas de atención e intervención psicosocial dirigidos a esta población.

En relación con los procesos de adaptación, se identificó que los desplazamientos de zonas rurales a zonas urbanas, el proceso de reunificación de las familias y la transición a un contexto de violencia armada organizada son los principales retos de niños y niñas excombatientes a la vida civil (Denov y Marchand, 2004). En este proceso, Denov y Marchand (2014) encontraron que lo que más impacta son las situaciones de rechazo y estigmatización en la que los menores experimentaron juicios comunitarios, desconfianza, discriminación y exclusión. A esto se le suman otras discriminaciones relacionadas con la etnia, la clase social o el género (Lugo, 2008).

En cuanto al componente afectivo, se encontró que esta población, presenta una afectación emocional relacionada con experiencias de sufrimiento y dolor (Guzmán, Villalba y Fernández, 2016). En las narrativas de mujeres excombatientes que fueron vinculadas cuando eran niñas, se identificaron dificultades para asumir o retomar un nuevo proyecto de vida porque sienten que no cuentan con protección, experimentan miedo y desconfianza (Ocampo et al., 2014), así como inseguridad en sí mismas (Chamorro, 2012). Adicionalmente, según Simbaqueba (2017) y Pinto (2009), las mujeres se enfrentan a estereotipos asociados con roles tradicionales de género, que deben asumir y reproducir tras la desvinculación.

Para Garcés y Chamorro (2011) es necesario considerar los modelos de relacionamiento, pues uno de los factores de vulnerabilidad, son los vínculos débiles, establecidos desde edades tempranas, y que con el tiempo, le generan a esta población miedos para vincularse afectivamente con otros y obstaculizan retomar los lazos afectivos con su familia de origen. En esta dimensión, el restablecimiento de la confianza es un proceso de configuración histórica e intersubjetiva determinante que puede convertirse en un medio para garantizar una atención coherente con las necesidades específicas de esta población (Gómez, Caicedo y Vallejo, 2011).

Otra categoría identificada fueron los cuestionamientos por la identidad. Lara y Delgado (2010) plantean que esta pregunta surge porque en la vivencia de la guerra se experimentan rupturas en sus subjetividades, ya que hay diferentes tiempos para la elaboración de duelos y para lograr restablecer sus vínculos. En discursos de jóvenes desmovilizados analizados por Jiménez-Gómez y Ramírez 
Loaiza (2016), se ha encontrado que valoran la posibilidad de ser actores significativos en el proceso de paz; y por ende, poder ejercer su ciudadanía. En sus narrativas, emergieron también cuestionamientos relacionados con el bienestar social, el vivir en sociedad, la identidad, el territorio y la responsabilidad; aspectos que desde los autores dan indicaciones de sus construcciones sobre sus subjetividades políticas.

Finalmente, frente a los programas de atención e intervención a esta población, se ha identificado la percepción de menores sobre estos programas, al igual que algunos logros, desafíos y dificultades. En relación con las percepciones, Molina (2011) realizó una investigación sobre cómo jóvenes desvinculados viven esta experiencia e identificaron que ellos valoran estos espacios como una forma de lograr beneficios económicos del Estado cuando sean mayores de edad, pero a la vez como una manera para resolver su situación jurídica. Los jóvenes entrevistados expresaron que no se consideraban víctimas y se definían como excombatientes. En su discurso no se hace referencia a que les fueron restituidos sus derechos y ellos no perciben mayores diferencias con el programa de adultos, a excepción de resolver la situación judicial y tener acceso a la educación (Simbaqueba, 2017).

En los logros se han encontrado programas que brindan intervención social, acompañamiento psicológico y restitución de derechos (Rojas, 2014). Se encuentra que la participación contribuye a que se generen transformaciones en los repertorios emocionales y sentimientos morales (Lara y Delgado, 2010). A través de diferentes iniciativas de trabajo grupal, terapéutico (Aguirre, 2014; Aguirre y Muñoz, 2011) y educativo (Alzate, 2009) se puede reactivar la capacidad relacional de jóvenes excombatientes.

En los desafíos, Simbaqueba (2017) hace énfasis en el término de "agencia" y concluye que los programas de reintegración no están orientados a generar habilidades para que cada uno de los niños sea capaz de tomar el rumbo de su propia vida. Al respecto,
Vera (2017) encuentra que las experiencias de los menores van más allá de las lógicas burocráticas y del enfoque de derechos y deberes. En este sentido, la experiencia de vida, según la autora, no puede reducirse a la experiencia del paso por la guerrilla y su entrada al ICBF, sino que existen otros eventos que también podrían ser foco de atención y, desde allí, podría asumirse la experiencia de vida y el reconocimiento de sus competencias.

Como dificultades, Villanueva y colaboradores (2017) encontraron que las organizaciones se encuentran limitadas en la implementación de los programas, siendo esto un riesgo de nuevos reclutamientos. Los programas son implementados en medio de tensiones relacionadas con la voluntad política, los acuerdos con los grupos armados, las crisis y dificultades de las relaciones locales, regionales y nacionales (Estrada, González, Diazgranados y Toro, 2016). Adicionalmente, sus marcos de referencia, al ser inspirados en experiencias internacionales, pueden producir violencias simbólicas en vez de cumplir con su deber de proteger (Vera, 2017). A esto se suma que, en ocasiones, los proyectos estan pensados para continuar la vida dentro de la ciudad, y no en las zonas rurales, además estos programas desconocen cómo trabajar con personas de etnias particulares (Simbaqueba, 2017).

\section{Recomendaciones}

De las investigaciones revisadas se han extraído algunas recomendaciones que pueden orientar las investigaciones y programas de atención psicosocial (Tabla 2).

Cada una de estas indicaciones hace patente la necesidad de reconocer a cada uno de los niños y jóvenes como un sujeto atravesado por una historia particular y no solo como un exponente más de un grupo mayor al que estaría subordinado. Las intervenciones deben evitar abordar a esta población como una masa homogénea, ya que esto podría llevar a desconocer las particularidades de cada participante. 
Tabla 2.

Recomendaciones para el trabajo con niños y niñas excombatientes

\begin{tabular}{|c|c|c|}
\hline Dimensión & Recomendación & Con base en \\
\hline $\begin{array}{l}\text { Representaciones so- } \\
\text { bre la infancia }\end{array}$ & $\begin{array}{l}\text { Reconocer construcciones de infancia desde una perspectiva } \\
\text { contextual. }\end{array}$ & Vera (2017) \\
\hline Capacidad de agencia & $\begin{array}{l}\text { Reconocer la capacidad de agencia de niños, niñas y jóvenes } \\
\text { en cada uno de los procesos y las posibles construcciones } \\
\text { sobre sí mismos. }\end{array}$ & $\begin{array}{l}\text { Moreno, Carmona, y Tobón } \\
\text { (2010) } \\
\text { Wainryb (2011) } \\
\text { Romero y Chávez (2008) }\end{array}$ \\
\hline $\begin{array}{l}\text { Reconocimiento de la } \\
\text { subjetividad e inter- } \\
\text { subjetividad }\end{array}$ & $\begin{array}{l}\text { Comprender las subjetividades que han construido como } \\
\text { guerreros “la zona de vacío que representa la condición de } \\
\text { víctima-victimario" y las relaciones con la socialización po- } \\
\text { lítica. } \\
\text { Interpretar la pretensión de los jóvenes por un reconoci- } \\
\text { miento intersubjetivo. }\end{array}$ & $\begin{array}{l}\text { Aguirre y Muñoz, (2011) } \\
\text { Amador-Baquiro, (2010) } \\
\text { Jiménez Gómez y Ramírez } \\
\text { Loaiza (2016) } \\
\text { Bejarano-Sanadria, y Delga- } \\
\text { do-Salazar, (2017) }\end{array}$ \\
\hline $\begin{array}{l}\text { Afectaciones más allá } \\
\text { de la perspectiva de } \\
\text { trauma }\end{array}$ & $\begin{array}{l}\text { Reconocer las implicaciones de la participación en el conflic- } \\
\text { to armado en el razonamiento moral. } \\
\text { Comprender la forma en que el conflicto armado afecta los } \\
\text { procesos de constitución de la identidad. }\end{array}$ & $\begin{array}{l}\text { Vera (2017) } \\
\text { Cifuentes (2008) }\end{array}$ \\
\hline $\begin{array}{l}\text { Abordaje desde las } \\
\text { narrativas }\end{array}$ & $\begin{array}{l}\text { Considerar la narratividad y la temporalidad de la experien- } \\
\text { cia, como un elemento reivindicador de esperanza, de me- } \\
\text { moria y de fragilidad humana. }\end{array}$ & $\begin{array}{l}\text { Torres (2015) } \\
\text { Romero y Chávez (2008) }\end{array}$ \\
\hline Análisis diferencial & $\begin{array}{l}\text { Realizar un análisis diferencial en términos de género, gene- } \\
\text { ración, etnia y territorio, entre otros. }\end{array}$ & $\begin{array}{l}\text { Cifuentes, Aguirre y Lugo } \\
\text { (2011). }\end{array}$ \\
\hline $\begin{array}{l}\text { La dimensión afectiva } \\
\text { en los procesos de re- } \\
\text { integración }\end{array}$ & $\begin{array}{l}\text { Crear escenarios que logren promover el vínculo y la con- } \\
\text { fianza con las comunidades, así como con las familias y los } \\
\text { contextos escolares. } \\
\text { Acompañar a la población para la construcción de relaciones } \\
\text { sociales basadas en el afecto. } \\
\text { Generar escenarios de reconocimiento del amor, del derecho } \\
\text { y la valoración social. }\end{array}$ & $\begin{array}{l}\text { Aguirre y Muñoz, (2011); } \\
\text { Chamorro, (2012); Garcés y } \\
\text { Chamorro (2011). } \\
\text { Bejarano-Sanadria, y Delga- } \\
\text { do-Salazar, (2017) }\end{array}$ \\
\hline El papel de la familia & $\begin{array}{l}\text { Redefinir el concepto de familia que vaya más allá de la vi- } \\
\text { sión tradicional. }\end{array}$ & $\begin{array}{l}\text { Cifuentes, Aguirre y Lugo } \\
\text { (2011) }\end{array}$ \\
\hline $\begin{array}{l}\text { Contexto rural y con- } \\
\text { texto urbano }\end{array}$ & $\begin{array}{l}\text { Contar con programas que incluyan la promoción de compe- } \\
\text { tencias para la reincorporación a la vida civil en los contex- } \\
\text { tos urbanos y rurales. }\end{array}$ & Pinto (2009) \\
\hline $\begin{array}{l}\text { Competencias ciuda- } \\
\text { danas }\end{array}$ & $\begin{array}{l}\text { Potenciar el trabajo en red entre los diferentes Hogares Tu- } \\
\text { tores para favorecer capacidades sociales y competencias } \\
\text { ciudadanas. } \\
\text { Reconocer a cada individuo como un sujeto con derechos y } \\
\text { responsabilidades. }\end{array}$ & $\begin{array}{l}\text { Vera (2017) } \\
\text { Pinto (2009) } \\
\text { Romero y Chávez (2008) }\end{array}$ \\
\hline $\begin{array}{l}\text { El rol del equipo psi- } \\
\text { cosocial }\end{array}$ & $\begin{array}{l}\text { Promover que los profesionales en los procesos de interven- } \\
\text { ción social logren posicionarse como sujetos de confianza } \\
\text { con los que se logre construir relaciones dialógicas. }\end{array}$ & Chamorro (2012) \\
\hline
\end{tabular}

Fuente: elaboración propia. 


\section{Conclusiones}

A partir de la revisión de la literatura sobre los procesos de vinculación, permanencia y reintegración de niños y niñas que participaron en grupos armados ilegales de Colombia (GAI) se encuentra que es un proceso en el que existen múltiples variables. Además, es un fenómeno que se encuentra enmarcado en una continua interacción entre los factores personales y contextuales. Es un fenómeno que con la aparición de nuevos actores criminales no sólo hace posible que continúe el reclutamiento de menores, sino que, además, dada la constante precariedad de las zonas rurales, algunos optan por la participación de los niños y las niñas en las cadenas de valor de las economías ilegales que manejan estos grupos.

Las perspectivas sobre las situaciones o motivaciones de vinculación de los niños y las niñas a los GAl son diversas. De las investigaciones revisadas se ha extraído que la forma en cómo los menores viven el proceso dista mucho del tiempo en el que se reconstruyen las narrativas sobre la experiencia. Es decir, si en el momento de la desmovilización siguen siendo niños o si ya son jóvenes o adultos, esto le otorga diferentes matices a las reconstrucciones e interpretaciones de la experiencia. A su vez, los investigadores han señalado variables importantes como la influencia del contexto y el funcionamiento familiar violento, así como la construcción de débiles vínculos afectivos en su desarrollo temprano.

Los programas de intervención psicosocial se despliegan como escenarios problemáticos, pues si bien acompañan en los procesos de transición a la vida civil, presentan a su vez numerosas dificultades. En Colombia, los niños aún siguen en riesgo de ser reclutados por grupos armados ilegales o bandas criminales y es necesario considerar que el fenómeno es dinámico, multidimensional y que el papel del Estado es fundamental para abordar muchos de los problemas de desarrollo estructural que contribuyen al sostenimiento de este fenómeno, tales como la pobreza, la falta de educación y la violencia intrafamiliar. Finalmente, en las investigaciones identificadas se evidencia un interés por recuperar las voces de los niños y las niñas que han vivido la experiencia de pertenecer a un grupo armado ilegal y las miradas abordan tanto la perspectiva de víctima como la de un actor social que cuenta con capacidad de agencia en su historia de vida.

\section{Referencias}

Aguirre, N. y Muñoz, C. (2011). De la Infancia a la Guerra: Una Continuidad Mental. Revista Eleuthera, 5, 51-76.

Aguirre, N. (2014). Después de la guerra: el individuo, el grupo y la relación de confianza. Tendencias y Retos, 19(2), 109-121.

Alzate, O. (2009). Las relaciones intrapersonales e interpersonales en estudiantes desvinculados del conflicto armado colombiano e incluidos en el aula del sol. Revista Luna Azul, 29, 25-31.

Amador-Baquiro, J. C. (2010). El intersticio de la víctima-victimario: un análisis de los procesos de subjetivación de cuatro desvinculados de grupos armados en Colombia. Universitas Humanística, (69), 163-184. https://doi. org/10.1016/j.ibiod.2013.01.001

Bácares, C. (2014). Los pequeños ejércitos: las representaciones sobre la vida y la muerte de los niños, niñas y jóvenes desvinculados de los grupos armados ilegales colombianos. Bogotá: Editorial Magisterio.

Bácares, C. (2015). Los niños, niñas y jóvenes desvinculados de los grupos armados ilegales en Colombia: ¿Víctimas de la violencia política o sujetos del delito? Estudios Socio-Jurídicos, 17(2), 233-262. https://doi.org/10.12804/ esj17.02.2015.07

Bácares, C. (2017). Siete tesis para una lectura multidimensional y en larga duración del reclutamiento ilícito de los niños, niñas y adolescentes en Colombia. Cuadernos de Marte, 8(12), 255316. https://doi.org/10.1515/BC.2007.140

Bejarano-Sanadria, C. y Delgado-Salazar, R. (2017). Reconocimiento de los jóvenes desmovilizados de los grupos armados en Colombia: transiciones y desafíos para las prácticas de orientación escolar. Magis: Revista Internacional 
de Investigación 9(19), 149-164. https://doi. org/10.11144/Javeriana.m9-19.rjdg

Bjørkhaug, I. (2010). Child Soldiers in Colombia: The Recruitment of Children into Non-state Violent Armed Groups. Research Working Paper, 27, Brighton: Microcon. https://doi.org/10.2139/ ssrn. 1650250

Blom, F. y Pereda, N. (2009). Niños y niñas soldado: Consecuencias psicológicas e intervención. Anuario de Psicología, 40(3), 329-344.

Caicedo, R. M. (2012). Los rastros del conflicto colombiano y las políticas para niñas, niños y jóvenes desvinculados de grupos armados. Trabajo Social, (14), 117-126. http://doi.org/10.15446/ts

Carmona, J. (2013). Las Niñas de la Guerra. Manizales: Universidad Católica de Manizales.

Carmona, J. A. y Tobón, J. F. (2007). Explicaciones del fenómeno de niñas soldados en Antioquia, Colombia: un análisis comparativo de la visión de las niñas desvinculadas de los Grupos Armados llegales y de los niños estudiantes de zonas rurales de Antioquia con un perfil psicosocial similar. Informe, (9), 41-53.

Carmona, J., Moreno, F. y Tobón, J. F. (2012). Child Soldiers in Colombia: Five Views. Universitas Psychologica, 11(3), 755-768. https://doi. org/10.11144/Javeriana.upsy11-3.cscf

Centro Nacional de Memoria Histórica [CNMH]. (2017). Una guerra sin edad: Informe Nacional de Reclutamiento y utilización de niños, niñas y adolescentes en el conflicto armado colombiano. Bogotá: $\mathrm{CNMH}$.

Chamorro, L. (2012). Los cautiverios de niñas y jóvenes excombatientes de grupos armados colombianos. Trabajo Social, 14, 127-145.

Cifuentes, M. (2008). El Sí y el Otro en la constitución de la identidad: niñas, niños y adolescentes desvinculados del conflicto armado. Trabajo Social, 10, 9-27.
Cifuentes, M., Aguirre, N. y Lugo, N. (2011). Niñas, Niños y Jóvenes Excombatientes: Revisión de Tema. Revista Eleuthera, 5, 93-124.

Correa, C., Jiménez, A. M., Ladisch, V. y Salazar, G. (2014). Reparación integradora para niños, niñas y jóvenes víctimas de reclutamiento ilícito en Colombia. Revisado en: https://www.ictj. org/sites/default/files/ICTJ-Informe-ColombiaReparacion-reclutamiento-ilegal-2014.pdf

Denov, M. y Marchand, I. (2014). "One cannot take away the stain": Rejection and stigma among former child soldiers in Colombia. Peace and Conflict: Journal of Peace Psychology, 20(4), 411-411. https://doi.org/10.1037/pac0000066

Downing, C. (2014). Child recruitment to illegal armed groups in Colombia: Peacebuilding and development challenges. Journal of Peacebuilding and Development, 9(3), 33-49. https://doi.org/10.1080/15423166.2014.984580

Estrada, Á., González, C., Diazgranados, S. y Toro, M. (2006). Atmósfera sociomoral y atención de los menores desvinculados del conflicto armado en Colombia. Revista Infancia Adolescencia y Familia, 1(2), 223-246.

Fajardo, M. A., Ramírez, M. P., Valencia, M. I. y Ospina-Alvarado, M. C. (2018). Más allá de la victimización de niñas y niños en contextos de conflicto armado: Potenciales para la construcción de paz. Universitas Psychologica, 17(1), 1-14. https://doi.org/10.11144/Javeriana. upsy17-1.mavn

Garcés, D. y Chamorro, L. (2011). Niñas, niños y jóvenes desvinculados del conflicto armado: ¿qué sucede con sus vínculos afectivos y con sus figuras de apego antes y durante su permanencia en los grupos armados? Revista Eleuthera, 5, 36-50.

Gómez, C. M., Caicedo, R. M. y Vallejo, S. Y. (2011). La confianza un proceso de configuración histórica e intersubjetiva en niñas, niños y jóvenes desvinculados del conflicto armado. Revista Eleuthera, 5, 77-92. 
Granados, Á. y Lavado, M. (2015). La reintegración de los niños, niñas y adolescentes víctimas de reclutamiento ilícito a la vida civil en los procesos de desvinculación (Tesis de Maestría). Universidad Militar Nueva Granada. Bogotá, Colombia.

Guzmán, K., Villalba, L. y Fernández, M. (2016). Emociones en niños y adolescentes desde la experiencia del desplazamiento y la vinculación a los grupos armados en Colombia. Panorama, 10(19), 85-96. http://doi.org/10.15765/pnrm.v10i19.834

Jiménez Gómez, K. L. y Ramírez Loaiza, V. (2016). Aproximaciones a la subjetividad y socialización política de jóvenes excombatientes del conflicto armado en Colombia. Trabajo Social, 18, 77-90.

Lara, L. y Delgado, R. (2010). Trasegar de las subjetividades y las memorias de las y los jóvenes desmovilizados en el tránsito a la vida civil. Una mirada a los programas educativos y de apoyo psicosocial. Universitas Humanística, 70, 29-56.

Lugo, V. (2018). Niños y jóvenes excombatientes en Colombia: ¿Por qué se vinculan y separan de la guerra? Athenea Digital, 18(2), 1-22. https:// doi.org/10.5565/rev/athenea.1933

Mesa, J. (2017). Hacia una nueva mirada de la reintegración de desmovilizados en Colombia: conceptos, enfoques y posibilidades. Revista CS, 23, 105-133. https://doi.org/10.18046/recs.i23.2437

Molina, G. P. (2011). La atención estatal a menores de edad desvinculados del conflicto armado: el Hogar José (Tesis de Maestría). Universidad Nacional de Colombia. Bogotá, Colombia. Recuperado de http://www.bdigital.unal. edu.co/6336/

Montoya, A. M. (2008). Niños y jóvenes en la guerra en Colombia. Aproximación a su reclutamiento y vinculación. Opinión Jurídica, 7(13), 37-51.

Moreno, F., Carmona, J. y Tobón, F. (2010). ¿Por qué se vinculan las niñas a los grupos guerrilleros y paramilitares en Colombia? Revista Latinoamericana de Psicología, 42(3), 453-467.
Nabuco, P. y Duarte, R. (2018). Child soldiers as peace-builders in Colombian peace talks between the government and the FARC-EP. Conflict, Security and Development, 18(5), 387-408. https://doi.org/10.1080/14678802.2018.1511164

Niño, N. (2016). Niños, niñas, jóvenes y grupos armados ilegales: experiencias de exclusión e inclusión social en América Latina. Argumentos. Revista de Crítica Social, (18), 177-206.

Ocampo, M., Baracaldo, P., Arboleda, L. y Escobar (2014). Relatos de vida de mujeres desmovilizadas: Análisis de sus perspectivas de vida. Informes Psicológicos, 14(1), 109-128.

Pieschacón, F., Melguizo, M. C. y González, P. (2006). Estudio exploratorio de patrones culturales que contribuyen a la vinculación de niños, niñas y jóvenes a los grupos armados en Colombia. Bogotá: Corporación Alotropía.

Pinto, E. (2009). Identidades y familias de jóvenes madres desvinculadas del conflicto armado. Trabajo Social, 11, 107-124.

Rojas, W. (2014). Programas de intervención social en Bogotá a niños, niñas y jóvenes desvinculados de los grupos armados ilegales en el conflicto armado colombiano. El caso de Justapaz y Enséñame a Pescar. (Tesis de Maestría). Universidad Nacional de Colombia. Bogotá, Colombia.

Romero, Y. y Chávez, Y. (2008). El juego de la guerra, niños, niñas y adolescentes en el conflicto armado en Colombia. Tabula Rasa, 8, 197-210. https://doi.org/10.1016/S0034-3617(02)80214-4

Sánchez, L., Rojas, L. y Taborda, P. (2015). La triada del conflicto armado colombiano: jóvenes heridos y cicatrizados en la subjetividad. Pontoe-Vírgula, 17, 105-137.

Simbaqueba, A. (2017). La reintegración de menores excombatientes no es un juego de niños-agencia y empoderamiento en los procesos de niños, niñas y adolescentes desvinculados del conflicto armado. Universidad de los Andes. Revisado en: https://repositorio.uniandes.edu.co/xmlui/ handle/1992/7977 
Springer, N. (2012). Prisioneros combatientes. Primer informe exploratorio sobre el uso de niños, niñas y adolescentes para los propósitos del conflicto armado en Colombia. Disponible en: https://www.colombiasoyyo.org/docs/resumen_informe_Mayanasa.pdf

Torres, E. (2015). Narratividad y tiempo: Niños y niñas desvinculados del conflicto armado colombiano. En: Lenguaje cultura e investigación (pp. 59-82). Bogotá: Universidad Distrital Francisco José de Caldas.

Torrado, M. C. (2002). Niños, niñas y conflicto armado en Colombia: una aproximación al Estado del arte 1990-2001. En M. Bello y S. Ceballos (Eds.), Conflicto armado, niñez y juventud: Una perspectiva psicosocial. Bogotá: Fundación Dos Mundos.

Unicef. (1997). Cape Town principles and best practices on the recruitment of children into the armed forces and on demobilization and social reintegration of child soldiers in Africa. Disponible en: http://www.unicef.org/emerg/ files/Cape_Town_Principles(1).pdf

Valencia, O. L. y Daza, M. F. (2010). Vinculación a grupos armados: un resultado del conflicto armado en Colombia. Revista Diversitas: Perspectivas en Psicología, 6(2), 429. https:// doi.org/10.15332/s1794-9998.2010.0002.14

Valencia-Suescún, M. I., Ramírez, M., Fajardo, M. A. y Ospina-Alvarado, M. C. (2015). De la afectación a nuevas posibilidades: niñas y niños en el conflicto armado colombiano. Revista Latinoamericana de Ciencias Sociales, Niñez y Juventud, 13(2), 1037-1050. https://doi.org/10 $.11600 / 1692715 x .13234251114$

Vargas, G. A. y Restrepo-Jaramillo, N. (2016). Child Soldiering in Colombia: Does Poverty Matter? Civil Wars, 18(4), 467-487. https://doi.org/10. 1080/13698249.2017.1297051

Velasco, F. y García, C. (2013). Menores desmovilizados de los grupos armados víctimas del conflicto. Línea de Derecho Penal del Grupo de Derecho Público. Universidad Militar Nueva Granada. Bogotá.

Vera, A. (2017). Del combate a la protección: Discursos, relatos de vida y prácticas estatales. (Tesis de Pregrado, Programa Antropología). Universidad del Rosario.

Villanueva, J., Loots, G., Losantos, Ma., Exeni, S., Berckmans, I. y Derluyn, I. (2017). Reinsertion processes of children disengaged from armed groups in Colombia: what is the problem represented to be? Revista Eleuthera, 16, 85-100. https://doi.org/10.17151/ eleu.2017.16.6.REINSERTION

Wainryb, C. (2011). 'And So They Ordered Me to Kill a Person': Conceptualizing the Impacts of Child Soldiering on the Development of Moral Agency. Human Development, 54. 273-300. https://doi.org/10.1159/000331482 